PROCEEDINGS OF THE

AMERICAN MATHEMATICAL SOCIETY

Volume 137, Number 10, October 2009, Pages 3533-3541

S 0002-9939(09)09906-7

Article electronically published on May 19, 2009

\title{
THE COMPLEX VOLUMES OF TWIST KNOTS
}

\author{
JINSEOK CHO, JUN MURAKAMI, AND YOSHIYUKI YOKOTA
}

(Communicated by Daniel Ruberman)

\begin{abstract}
For a given hyperbolic knot, the third author defined a function whose imaginary part gives the hyperbolic volume of the knot complement. We show that, for a twist knot, the function actually gives the complex volume of the knot complement using Zickert's and Neumann's theory of the extended Bloch groups and the complex volumes.
\end{abstract}

\section{INTRODUCTION}

In [1, Kashaev defined an invariant of a link and conjectured the following:

$$
\operatorname{vol}(L)=2 \pi \lim _{N \rightarrow \infty} \frac{\log \left|\langle L\rangle_{N}\right|}{N},
$$

where $L$ is a hyperbolic link, $\operatorname{vol}(L)$ is the hyperbolic volume of the link complement, $\langle L\rangle_{N}$ is the Kashaev invariant of $L$, and $N$ is the positive-integer variable. Afterwards, in [3], the complexified volume conjecture was proposed as follows and confirmed in some cases with numerical computations:

$$
i(\operatorname{vol}(L)+i \operatorname{cs}(L)) \equiv 2 \pi \lim _{N \rightarrow \infty} \frac{\log \langle L\rangle_{N}}{N}\left(\bmod \pi^{2}\right),
$$

where $\operatorname{cs}(L)$ is the Cheeger-Chern-Simons invariant of the hyperbolic link complement. We call $\operatorname{vol}(L)+i \operatorname{cs}(L)$ the complex volume of $L$.

On the other hand, the third author developed a special ideal triangulation of a hyperbolic knot complement in [6] and showed that this triangulation has a close relation with the Kashaev invariant. He also defined a function $V$ from this triangulation and showed that, after evaluating $V$ with the geometric solution of the hyperbolicity equations, the imaginary part of $V$ becomes the volume of the knot complement.

Therefore, it is natural to expect that, after evaluating, the real part of $V$ becomes the Cheeger-Chern-Simons invariant of the knot complement modulo $\pi^{2}$. In this article, we show such a coincidence for the twist knots using Zickert's and Neumann's theory of the extended Bloch groups and the complex volumes in [7] and [4.

Received by the editors December 29, 2008.

2000 Mathematics Subject Classification. Primary 57M27; Secondary 51M25, 58J28.

Key words and phrases. Twist knot, volume conjecture, complex volume.

This work was carried out while the first author was visiting Waseda University with the support of the Korea National Foundation Grant funded by the Korean Government (MOEHRD) (KRF-2007-612-C00037).

(C)2009 American Mathematical Society Reverts to public domain 28 years from publication 


\section{Preliminaries}

For a positive integer $n$, let $T_{n}$ be the twist knot with $n+4$ crossings as in Figure 1. We label some of the crossings with integers $0,1, \ldots, n+1$ as in Figure 1. Also consider a $(1,1)$-tangle diagram whose closure is $T_{n}$. Following the method in [6], we assign a complex variable $z_{k}$ to the edge of $T_{n}$ that connects the over-crossing point of the $k$ th crossing and the under-crossing point of the $(k+1)$ th crossing. (See Figure 1. The arcs around the crossing points represent the surviving tetrahedra after the collapsing process, which will be briefly mentioned after Figure 2.)

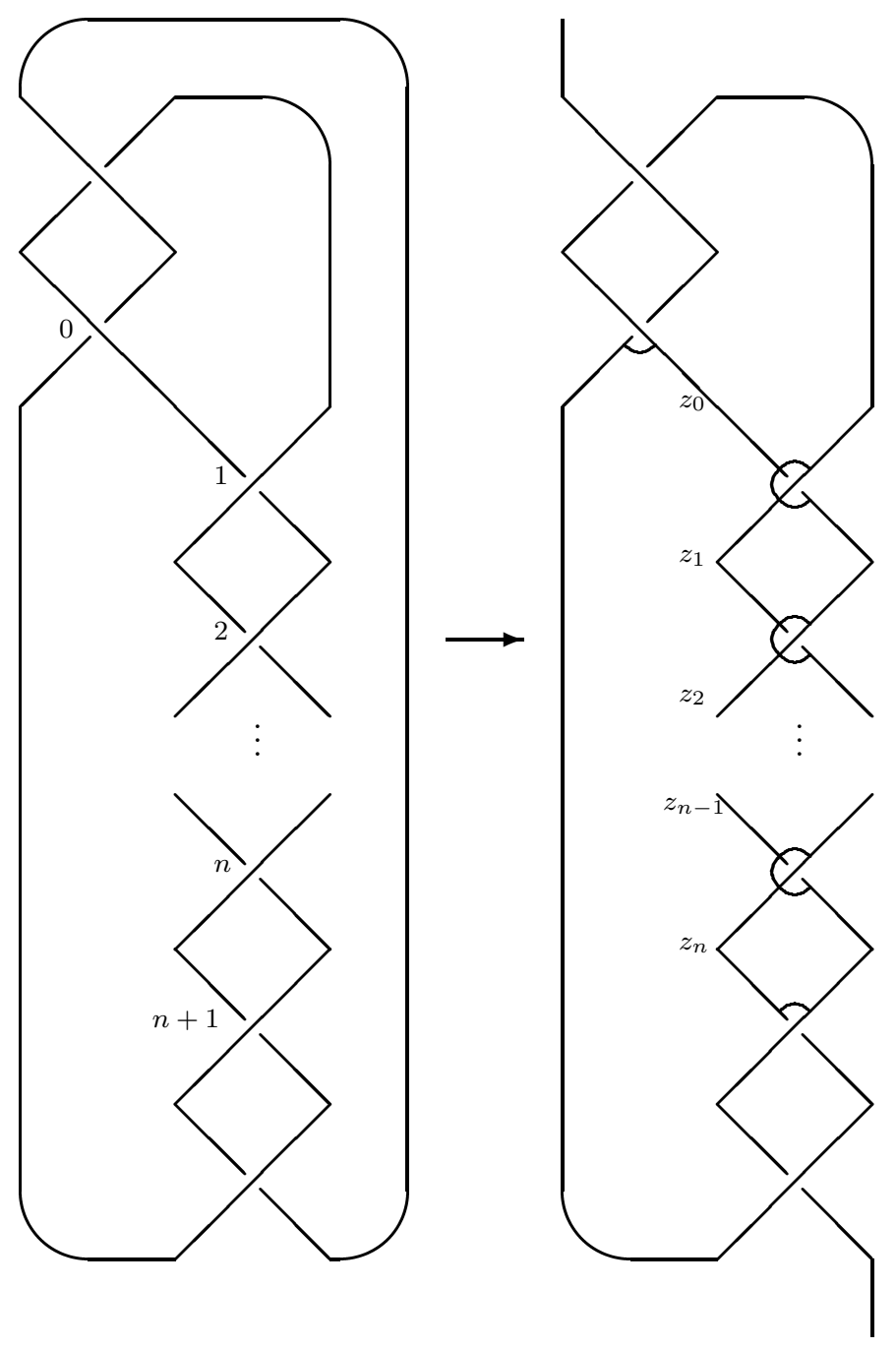

Figure 1 

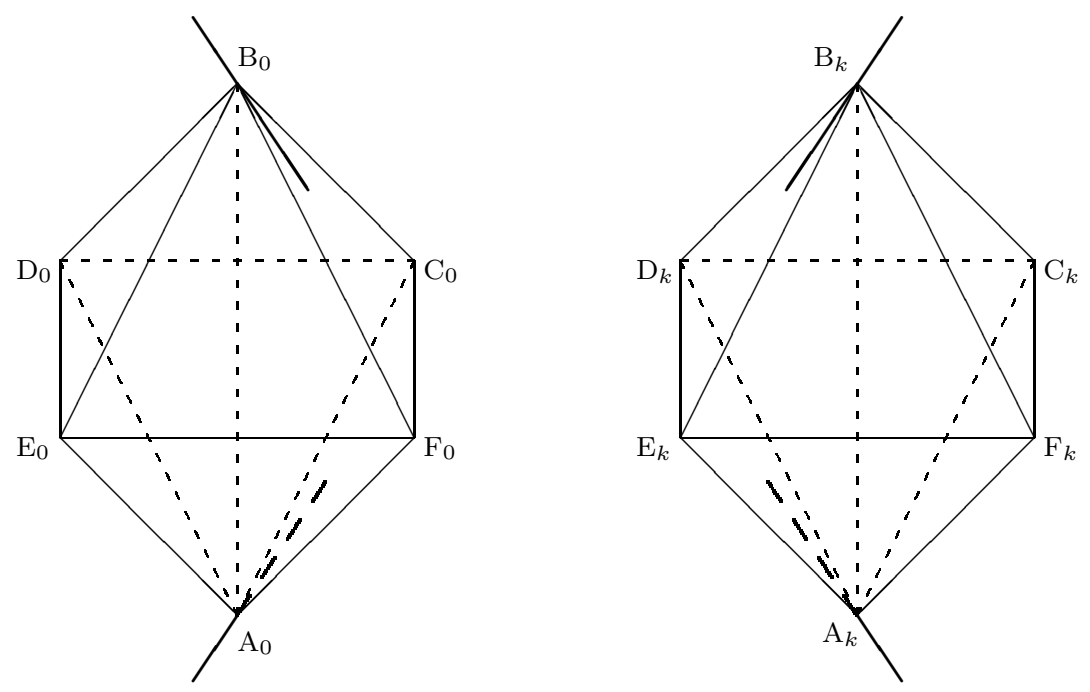

FiguRE 2

Let $I=\{1,2, \ldots, n\}$. We put an octahedron $\mathrm{A}_{k} \mathrm{~B}_{k} \mathrm{C}_{k} \mathrm{D}_{k} \mathrm{E}_{k} \mathrm{~F}_{k}$ at the $k$ th crossing of the tangle diagram for $k \in I \cup\{0, n+1\}$ so that $\mathrm{A}_{k} \mathrm{~B}_{k}$ is perpendicular to the plane; $\mathrm{A}_{k}$ and $\mathrm{B}_{k}$ touch the under-crossing point and the over-crossing point respectively; and $\mathrm{C}_{k}, \mathrm{D}_{k}, \mathrm{E}_{k}, \mathrm{~F}_{k}$ are to be the northeast, northwest, southwest, and southeast respectively. (See Figure 2.) Note that the octahedron $\mathrm{A}_{k} \mathrm{~B}_{k} \mathrm{C}_{k} \mathrm{D}_{k} \mathrm{E}_{k} \mathrm{~F}_{k}$ splits into 4 tetrahedra: in our case, $\mathrm{A}_{k} \mathrm{~B}_{k} \mathrm{C}_{k} \mathrm{D}_{k}, \mathrm{~A}_{k} \mathrm{~B}_{k} \mathrm{D}_{k} \mathrm{E}_{k}, \mathrm{~A}_{k} \mathrm{~B}_{k} \mathrm{E}_{k} \mathrm{~F}_{k}$, and $\mathrm{A}_{k} \mathrm{~B}_{k} \mathrm{~F}_{k} \mathrm{C}_{k}$.

We identify the edges $\mathrm{A}_{0} \mathrm{D}_{0}$ with $\mathrm{A}_{0} \mathrm{~F}_{0}, \mathrm{~B}_{0} \mathrm{C}_{0}$ with $\mathrm{B}_{0} \mathrm{E}_{0}, \mathrm{~A}_{k} \mathrm{C}_{k}$ with $\mathrm{A}_{k} \mathrm{E}_{k}$, and $\mathrm{B}_{k} \mathrm{D}_{k}$ with $\mathrm{B}_{k} \mathrm{~F}_{k}$ respectively for $k \in I \cup\{n+1\}$. To obtain the ideal triangulation of the knot complement, we collapse some of the tetrahedra and glue the surviving tetrahedra together following the knot diagram. (For details, see [2] or 6.) In our case, we can obtain the ideal triangulation of the knot complement by gluing the $3 n+2$ tetrahedra $\mathrm{A}_{0} \mathrm{~B}_{0} \mathrm{E}_{0} \mathrm{~F}_{0}, \mathrm{~A}_{k} \mathrm{~B}_{k} \mathrm{C}_{k} \mathrm{D}_{k}, \mathrm{~A}_{k} \mathrm{~B}_{k} \mathrm{D}_{k} \mathrm{E}_{k}, \mathrm{~A}_{k} \mathrm{~B}_{k} \mathrm{E}_{k} \mathrm{~F}_{k}$, and $\mathrm{A}_{n+1} \mathrm{~B}_{n+1} \mathrm{C}_{n+1} \mathrm{D}_{n+1}$ for $k \in I$.

To apply the method in [6] to this triangulation, we parameterize each ideal tetrahedra with complex numbers as follows. Assign $z_{0}$ to the edge $\mathrm{A}_{0} \mathrm{~B}_{0}$ of $\mathrm{A}_{0} \mathrm{~B}_{0} \mathrm{E}_{0} \mathrm{~F}_{0}$, $z_{k-1}$ to the edge $\mathrm{A}_{k} \mathrm{~B}_{k}$ of $\mathrm{A}_{k} \mathrm{~B}_{k} \mathrm{C}_{k} \mathrm{D}_{k}, \frac{z_{k}}{z_{k-1}}$ to the edge $\mathrm{A}_{k} \mathrm{~B}_{k}$ of $\mathrm{A}_{k} \mathrm{~B}_{k} \mathrm{D}_{k} \mathrm{E}_{k}, \frac{1}{z_{k}}$ to the edge $\mathrm{A}_{k} \mathrm{~B}_{k}$ of $\mathrm{A}_{k} \mathrm{~B}_{k} \mathrm{E}_{k} \mathrm{~F}_{k}$, and $z_{n}$ to the edge $\mathrm{A}_{n+1} \mathrm{~B}_{n+1}$ of $\mathrm{A}_{n+1} \mathrm{~B}_{n+1} \mathrm{C}_{n+1} \mathrm{D}_{n+1}$ respectively for $k \in I$. Then, from [5] or [6], the hyperbolicity equations of the knot complement become

$$
\begin{aligned}
1-\frac{z_{0}}{z_{1}} & =\left(1-z_{0}\right)\left(1-\frac{1}{z_{0}}\right) \\
\left(1-\frac{z_{k}}{z_{k+1}}\right)\left(1-\frac{1}{z_{k}}\right) & =\left(1-z_{k}\right)\left(1-\frac{z_{k-1}}{z_{k}}\right) \quad \text { for } k=1,2, \ldots, n-1, \\
1-\frac{1}{z_{n}} & =\left(1-z_{n}\right)\left(1-\frac{z_{n-1}}{z_{n}}\right) .
\end{aligned}
$$


Note that the solution of these equations which induces the complete hyperbolic structure of the knot complement exists uniquely by virtue of the Lemma 2.3 of [6] . In this article, the unique solution is called the geometric solution.

Now let $r_{k}$ be the even integer satisfying the following equations for $k \in I \cup\{0\}$ :

$$
\begin{aligned}
& r_{0} \pi i=\log \left(1-\frac{z_{0}}{z_{1}}\right)-\log \left(1-z_{0}\right)-\log \left(1-\frac{1}{z_{0}}\right), \\
& r_{k} \pi i=\log \left(1-\frac{z_{k}}{z_{k+1}}\right)+\log \left(1-\frac{1}{z_{k}}\right)-\log \left(1-z_{k}\right)-\log \left(1-\frac{z_{k-1}}{z_{k}}\right) \\
& \quad \quad \text { for } k=1,2, \ldots, n-1, \\
& r_{n} \pi i=\log \left(1-\frac{1}{z_{n}}\right)-\log \left(1-z_{n}\right)-\log \left(1-\frac{z_{n-1}}{z_{n}}\right) .
\end{aligned}
$$

Then we can determine the $V$ function, following [6], as

$$
\begin{aligned}
& V\left(z_{0}, z_{1}, \ldots, z_{n}\right)=-\sum_{k \in I \cup\{0\}} r_{k} \pi i \log z_{k}+\left(\frac{\pi^{2}}{6}-\operatorname{Li}_{2}\left(\frac{1}{z_{0}}\right)\right) \\
& \quad+\sum_{k \in I}\left\{\left(\operatorname{Li}_{2}\left(z_{k-1}\right)-\frac{\pi^{2}}{6}\right)+\left(\frac{\pi^{2}}{6}-\operatorname{Li}_{2}\left(\frac{z_{k-1}}{z_{k}}\right)\right)+\left(\operatorname{Li}_{2}\left(\frac{1}{z_{k}}\right)-\frac{\pi^{2}}{6}\right)\right\} \\
& \quad+\left(\operatorname{Li}_{2}\left(z_{n}\right)-\frac{\pi^{2}}{6}\right) .
\end{aligned}
$$

With these notations, we state the main theorem of this article.

Theorem 2.1. Let $z_{0}, z_{1}, \ldots, z_{n}$ be the geometric solution of (2.1), which induces the complete hyperbolic structure of the $T_{n}$ complement. Then

$$
V\left(z_{0}, z_{1}, \ldots, z_{n}\right) \equiv i\left(\operatorname{vol}\left(T_{n}\right)+i \operatorname{cs}\left(T_{n}\right)\right)\left(\bmod \pi^{2}\right) .
$$

We will prove this theorem in Section 3 .

The main technique of the proof is Zickert's article [7. To apply it, we need the vertex ordering of each tetrahedron. We equip the vertex ordering according to the order of the vertices as follows: $\mathrm{B}_{0} \mathrm{~A}_{0} \mathrm{~F}_{0} \mathrm{E}_{0}, \mathrm{~A}_{1} \mathrm{~B}_{1} \mathrm{D}_{1} \mathrm{C}_{1}, \mathrm{~A}_{k} \mathrm{~B}_{k} \mathrm{C}_{k} \mathrm{D}_{k}$ for $k=2,3, \ldots, n+1, \mathrm{~A}_{k} \mathrm{~B}_{k} \mathrm{E}_{k} \mathrm{D}_{k}$ for $k \in I$, and $\mathrm{A}_{k} \mathrm{~B}_{k} \mathrm{E}_{k} \mathrm{~F}_{k}$ for $k \in I$. For example, the vertices $\mathrm{A}_{k}, \mathrm{~B}_{k}, \mathrm{E}_{k}, \mathrm{D}_{k}$ of the tetrahedron $\mathrm{A}_{k} \mathrm{~B}_{k} \mathrm{E}_{k} \mathrm{D}_{k}$ have the order $0,1,2$, 3 respectively.

Note that the vertex ordering of each tetrahedron induces orientations of edges and the tetrahedron. The induced orientation can be different from the original orientation induced by the knot complement. In our case, such a situation arises for $\mathrm{A}_{1} \mathrm{~B}_{1} \mathrm{D}_{1} \mathrm{C}_{1}$ and $\mathrm{A}_{k} \mathrm{~B}_{k} \mathrm{E}_{k} \mathrm{D}_{k}$ for $k \in I$.

To apply the main technique, we need the following lemma, which is induced directly from the gluing pattern of the triangulation.

Lemma 2.2. The ideal triangulation of the $T_{n}$ complement given as above is in concordance with the ordering of each edge which is induced by the vertex ordering defined above. In other words, if two edges are glued together by the triangulation, then the orientations of two edges induced by each vertex ordering coincide.

Now we will determine the element of the extended Bloch group $\widehat{\mathcal{B}}(\mathbb{C})$ corresponding to each tetrahedron using the method of [7]. At first, let $J=\{1,2,3\}$ 
and define a sign function $\sigma_{k}^{(j)}$ as

$$
\sigma_{k}^{(j)}= \begin{cases}-1 & \text { if } j=2 \text { or }(k, j)=(1,1) \\ 1 & \text { otherwise }\end{cases}
$$

for $k \in I \cup\{0, n+1\}$ and $j \in J$ except $(k, j)=(0,1),(0,2),(n+1,2),(n+1,3)$. Also let

$$
\begin{gathered}
\sigma_{0}^{(3)}\left[z_{0}^{(3)} ; p_{0}^{(3)}, q_{0}^{(3)}\right], \sigma_{1}^{(1)}\left[z_{1}^{(1)} ; p_{1}^{(1)}, q_{1}^{(1)}\right], \sigma_{k}^{(1)}\left[z_{k}^{(1)} ; p_{k}^{(1)}, q_{k}^{(1)}\right] \text { for } k \neq 1 \\
\sigma_{k}^{(2)}\left[z_{k}^{(2)} ; p_{k}^{(2)}, q_{k}^{(2)}\right], \sigma_{k}^{(3)}\left[z_{k}^{(3)} ; p_{k}^{(3)}, q_{k}^{(3)}\right], \text { and } \sigma_{n+1}^{(1)}\left[z_{n+1}^{(1)} ; p_{n+1}^{(1)}, q_{n+1}^{(1)}\right]
\end{gathered}
$$

be the elements of $\widehat{\mathcal{B}}(\mathbb{C})$ corresponding to the tetrahedra $\mathrm{B}_{0} \mathrm{~A}_{0} \mathrm{~F}_{0} \mathrm{E}_{0}, \mathrm{~A}_{1} \mathrm{~B}_{1} \mathrm{D}_{1} \mathrm{C}_{1}$, $\mathrm{A}_{k} \mathrm{~B}_{k} \mathrm{C}_{k} \mathrm{D}_{k}, \mathrm{~A}_{k} \mathrm{~B}_{k} \mathrm{E}_{k} \mathrm{D}_{k}, \mathrm{~A}_{k} \mathrm{~B}_{k} \mathrm{E}_{k} \mathrm{~F}_{k}$, and $\mathrm{A}_{n+1} \mathrm{~B}_{n+1} \mathrm{C}_{n+1} \mathrm{D}_{n+1}$ for $k \in I$ respectively. Then, by the definition above and the parameters of the ideal tetrahedra,

$$
\begin{array}{r}
z_{0}^{(3)}=z_{0}, z_{1}^{(1)}=\frac{1}{z_{0}}, z_{k}^{(1)}=z_{k-1} \text { for } k \neq 1, \\
z_{k}^{(2)}=\frac{z_{k-1}}{z_{k}}, z_{k}^{(3)}=\frac{1}{z_{k}}, \text { and } z_{n+1}^{(1)}=z_{n},
\end{array}
$$

for $k \in I$. To determine the integers $p_{k}^{(j)}$ and $q_{k}^{(j)}$ for $k \in I \cup\{0, n+1\}$ and $j \in J$, we use the following definition from (3.5) of [7. For a tetrahedron with a vertex ordering,

$$
\begin{aligned}
p \pi i+\log z & =\log c\left(g_{03}\right)+\log c\left(g_{12}\right)-\log c\left(g_{02}\right)-\log c\left(g_{13}\right), \\
q \pi i-\log (1-z) & =\log c\left(g_{02}\right)+\log c\left(g_{13}\right)-\log c\left(g_{01}\right)-\log c\left(g_{23}\right),
\end{aligned}
$$

where $[z ; p, q]$ is the element of $\widehat{\mathcal{B}}(\mathbb{C})$ corresponding to the ideal tetrahedron and $c\left(g_{a b}\right)$ is the complex number assigned to the edge connecting the ath and $b$ th vertex.

Zickert gave an explicit method for calculating the complex numbers in [7, but, in this article, we do not need the exact numbers. What we need is only the fact that if two edges are identified by the gluing pattern of the ideal triangulation, then the numbers assigned to these identified edges are the same. Using this fact, the following lemma is directly deduced from the gluing pattern of the triangulation.

Lemma 2.3. Let $\alpha_{k}, \beta_{k}, \gamma_{k}, \gamma_{k+1}, m_{k}$, and $\delta$ be the complex numbers assigned to the edges $\mathrm{B}_{k} \mathrm{E}_{k}, \mathrm{~B}_{k} \mathrm{~F}_{k}, \mathrm{C}_{k} \mathrm{D}_{k}, \mathrm{E}_{k} \mathrm{~F}_{k}, \mathrm{~A}_{k} \mathrm{~B}_{k}$, and $\mathrm{D}_{k} \mathrm{E}_{k}$ respectively for $k \in I$. Also let $\alpha_{0}$ and $\beta_{0}$ be the complex numbers assigned to the edges $\mathrm{B}_{0} \mathrm{~F}_{0}$ and $\mathrm{B}_{0} \mathrm{E}_{0}$ respectively. Then we can determine all the assigned complex numbers of each edge using $\alpha_{k}, \beta_{k}, \gamma_{k}, m_{k}, \delta, \alpha_{0}, \beta_{0}$, and $\gamma_{n+1}$ for $k \in I$ as in Figure 3. 


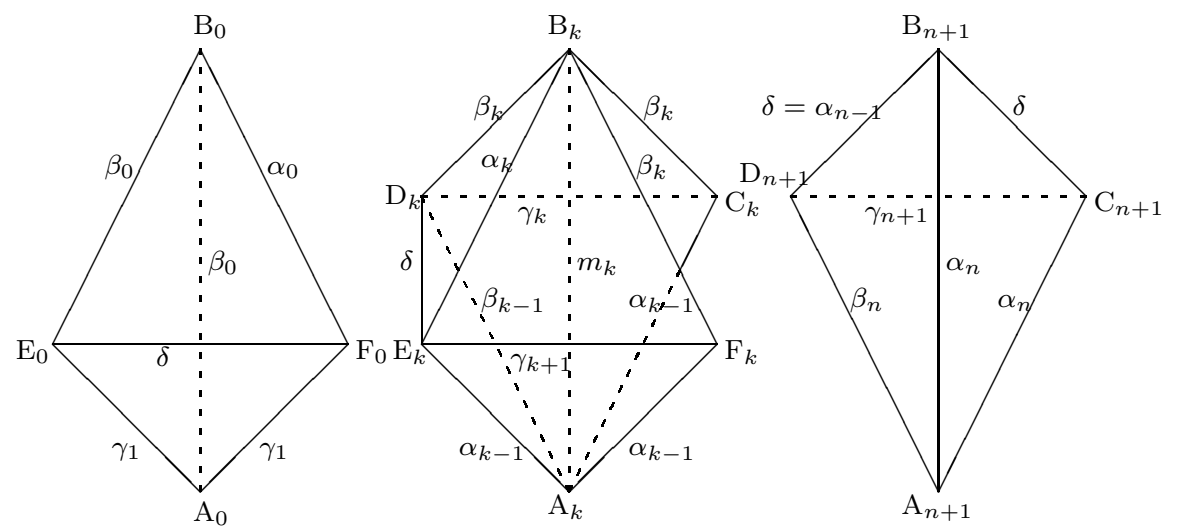

FiguRE 3

The following lemma is a direct consequence of (2.5), Lemma 2.2] and 4] and [7.

Lemma 2.4. Let $z_{0}, z_{1}, \ldots, z_{n}$ be the geometric solution of (2.1) and let $\sigma_{k}^{(j)}\left[z_{k}^{(j)} ; p_{k}^{(j)}, q_{k}^{(j)}\right]$ be the element of the extended Bloch group $\widehat{\mathcal{B}}(\mathbb{C})$ defined in (2.4), (2.5), and (2.6). Then

$$
i\left(\operatorname{vol}\left(T_{n}\right)+i \operatorname{cs}\left(T_{n}\right)\right) \equiv \sum_{\substack{k \in I \cup\{0, n+1\} \\ j \in J}} \sigma_{k}^{(j)} \widehat{L}\left[z_{k}^{(j)} ; p_{k}^{(j)}, q_{k}^{(j)}\right]\left(\bmod \pi^{2}\right),
$$

where $\widehat{L}[z ; p, q]=\operatorname{Li}_{2}(z)+\frac{1}{2} \log z \log (1-z)+\frac{\pi i}{2}(q \log z+p \log (1-z))-\frac{\pi^{2}}{6}$ is the extended dilogarithm function defined in $\widehat{\mathcal{B}}(\mathbb{C})$.

To prove the main theorem, we prove the coincidence of the right side of (2.3) and the right side of (2.9) in the next section.

\section{Proof of the Theorem 2.1}

To simplify the calculation, we define $\hat{p}_{k}^{(j)}$ by

$$
\hat{p}_{k}^{(j)} \pi i=p_{k}^{(j)} \pi i+\log z_{k}^{(j)}
$$

for $k \in I \cup\{0, n+1\}$ and $j \in J$. Then, the right side of (2.9) becomes

$$
\begin{aligned}
& \sum_{\substack{k \in I \cup\{0, n+1\} \\
j \in J}} \sigma_{k}^{(j)}\left\{\operatorname{Li}_{2}\left(z_{k}^{(j)}\right)-\frac{\pi^{2}}{6}\right\} \\
& \quad+\frac{\pi i}{2} \sum_{\substack{k \in I \cup\{0, n+1\} \\
j \in J}} \sigma_{k}^{(j)}\left\{q_{k}^{(j)} \log z_{k}^{(j)}+\hat{p}_{k}^{(j)} \log \left(1-z_{k}^{(j)}\right)\right\} \\
& =V\left(z_{0}, z_{1}, \ldots, z_{n}\right)+\sum_{k \in I \cup\{0\}} r_{k} \pi i \log z_{k} \\
& \quad+\frac{\pi i}{2} \sum_{\substack{k \in I \cup\{0, n+1\} \\
j \in J}} \sigma_{k}^{(j)}\left\{q_{k}^{(j)} \log z_{k}^{(j)}+\hat{p}_{k}^{(j)} \log \left(1-z_{k}^{(j)}\right)\right\} .
\end{aligned}
$$


Therefore, it is enough to show that

$$
\begin{array}{r}
\sum_{\substack{k \in I \cup\{0, n+1\} \\
j \in J}} \sigma_{k}^{(j)} \pi i\left\{q_{k}^{(j)} \log z_{k}^{(j)}+\hat{p}_{k}^{(j)} \log \left(1-z_{k}^{(j)}\right)\right\} \\
+2\left\{\sum_{k \in I \cup\{0\}} r_{k} \pi i \log z_{k}\right\} \equiv 0\left(\bmod 2 \pi^{2}\right) .
\end{array}
$$

\section{Lemma 3.1.}

$$
\begin{aligned}
r_{0} \pi i= & \left(q_{1}^{(2)}-q_{0}^{(3)}-q_{1}^{(1)}\right) \pi i, \\
r_{k} \pi i= & \left(q_{k}^{(3)}-q_{k}^{(2)}+q_{k+1}^{(2)}-q_{k+1}^{(1)}\right) \pi i \\
& \quad \text { for } k=1,2, \ldots, n-1, \\
r_{n} \pi i= & \left(q_{n}^{(3)}-q_{n+1}^{(1)}-q_{n}^{(2)}\right) \pi i .
\end{aligned}
$$

Proof. From Lemma 2.3 and (2.8), we obtain

$$
\begin{aligned}
q_{1}^{(1)} \pi i= & \log \left(1-\frac{1}{z_{0}}\right)+\log \beta_{0}+\log \beta_{1}-\log \gamma_{1}-\log m_{1}, \\
q_{k+1}^{(1)} \pi i= & \log \left(1-z_{k}\right)+\log \alpha_{k}+\log \beta_{k+1}-\log \gamma_{k+1}-\log m_{k+1} \\
& \quad \text { for } k=1,2, \ldots, n-1, \\
q_{n+1}^{(1)} \pi i= & \log \left(1-z_{n}\right)+\log \alpha_{n-1}-\log \gamma_{n+1}, \\
q_{k}^{(2)} \pi i= & \log \left(1-\frac{z_{k-1}}{z_{k}}\right)+\log \alpha_{k-1}+\log \beta_{k}-\log \delta-\log m_{k} \text { for } k \in I, \\
q_{0}^{(3)} \pi i= & \log \left(1-z_{0}\right)+\log \alpha_{0}+\log \gamma_{1}-\log \beta_{0}-\log \delta, \\
q_{k}^{(3)} \pi i= & \log \left(1-\frac{1}{z_{k}}\right)+\log \alpha_{k-1}+\log \beta_{k}-\log \gamma_{k+1}-\log m_{k} \text { for } k \in I .
\end{aligned}
$$

Applying these equations and $\delta=\alpha_{n-1}$ to (2.2), we obtain the result.

\section{Lemma 3.2.}

$$
\begin{aligned}
\sum_{\substack{k \in I \cup\{0, n+1\} \\
j \in J}} \sigma_{k}^{(j)} \hat{p}_{k}^{(j)} \pi i \log \left(1-z_{k}^{(j)}\right) & \equiv \sum_{\substack{k \in I \cup\{0, n+1\} \\
j \in J}} \sigma_{k}^{(j)} q_{k}^{(j)} \pi i \log z_{k}^{(j)} \\
& \equiv-\sum_{k \in I \cup\{0\}} r_{k} \pi i \log z_{k}\left(\bmod 2 \pi^{2}\right) .
\end{aligned}
$$

Proof. From (2.7) and Lemma 2.3, we know that

$$
\begin{aligned}
\hat{p}_{1}^{(1)} \pi i & =\log \alpha_{0}-\log \beta_{0}, \\
\hat{p}_{k+1}^{(1)} \pi i & =-\log \alpha_{k}+\log \beta_{k}, \\
\hat{p}_{k}^{(2)} \pi i & =\log \alpha_{k}-\log \beta_{k}-\log \alpha_{k-1}+\log \beta_{k-1}, \\
\hat{p}_{0}^{(3)} \pi i & =-\log \alpha_{0}+\log \beta_{0}, \\
\hat{p}_{k}^{(3)} \pi i & =\log \alpha_{k}-\log \beta_{k},
\end{aligned}
$$


for $k \in I$. Using these equations, (2.2), and Lemma 3.1, we can finish the proof as follows:

$$
\begin{aligned}
& \sum_{\substack{k \in I \cup\{0, n+1\} \\
j \in J}} \sigma_{k}^{(j)} \hat{p}_{k}^{(j)} \pi i \log \left(1-z_{k}^{(j)}\right) \\
=- & \hat{p}_{1}^{(1)} \pi i \log \left(1-\frac{1}{z_{0}}\right)+\sum_{k \in I} \hat{p}_{k+1}^{(1)} \pi i \log \left(1-z_{k}\right) \\
& \quad-\sum_{k \in I} \hat{p}_{k}^{(2)} \pi i \log \left(1-\frac{z_{k-1}}{z_{k}}\right)+\hat{p}_{0}^{(3)} \pi i \log \left(1-z_{0}\right)+\sum_{k \in I} \hat{p}_{k}^{(3)} \pi i \log \left(1-\frac{1}{z_{k}}\right) \\
=\left(-\log \alpha_{0}+\log \beta_{0}\right)\left\{\log \left(1-\frac{1}{z_{0}}\right)-\log \left(1-\frac{z_{0}}{z_{1}}\right)+\log \left(1-z_{0}\right)\right\} & \left.\quad+\log \left(1-\frac{z_{k}}{z_{k+1}}\right)+\log \left(1-\frac{1}{z_{k}}\right)\right\} \\
& +\sum_{k=1}^{n-1}\left(\log \alpha_{k}-\log \beta_{k}\right)\left\{-\log \left(1-z_{k}\right)-\log \left(1-\frac{z_{k-1}}{z_{k}}\right)\right. \\
& +\left(\log \alpha_{n}-\log \beta_{n}\right)\left\{-\log \left(1-z_{n}\right)-\log \left(1-\frac{z_{n-1}}{z_{n}}\right)+\log \left(1-\frac{1}{z_{n}}\right)\right\} \\
\equiv- & \sum_{k \in I \cup\{0\}} r_{k} \pi i \log z_{k}=-\left(q_{1}^{(2)}-q_{0}^{(3)}-q_{1}^{(1)}\right) \pi i \log z_{0} \\
& -\sum_{k=1}^{n-1}\left(q_{k}^{(3)}-q_{k}^{(2)}+q_{k+1}^{(2)}-q_{k+1}^{(1)}\right) \pi i \log z_{k}-\left(q_{n}^{(3)}-q_{n+1}^{(1)}-q_{n}^{(2)}\right) \pi i \log z_{n} \\
\equiv & \sum_{k \in I \cup\{0, n+1\}} \sigma_{k}^{(j)} q_{k}^{(j)} \pi i \log z_{k}^{(j)}\left(\bmod 2 \pi^{2}\right) .
\end{aligned}
$$

Applying Lemma 3.2 to (3.1), the proof of the theorem is finished.

\section{REFERENCES}

1. R. Kashaev, The hyperbolic volume of knots from the quantum dilogarithm, Lett. Math. Phys. 39 (1997), no. 3, 269-275. MR.1434238 (98b:57012)

2. H. Murakami, Kashaev's invariant and the volume of a hyperbolic knot after Y. Yokota, Physics and combinatorics 1999 (Nagoya), 244-272, World Sci. Publ., River Edge, NJ, 2001. MR.1865040(2002k:57031)

3. H. Murakami, J. Murakami, M. Okamoto, T. Takata, and Y. Yokota, Kashaev's conjecture and the Chern-Simons invariants of knots and links, Experiment. Math. 11 (2002), no. 3, 427-435. MR.1959752(2004a:57016)

4. W. Neumann, Extended Bloch group and the Cheeger-Chern-Simons class, Geom. Topol. 8 (2004), 413-474. MR2033484 (2005e:57042)

5. Y. Yokota, From the Jones polynomial to the A-polynomial of hyperbolic knots, Interdiscip. Inform. Sci. 9 (2003), no. 1, 11-21. MR2023102 (2004j:57014)

6. Y. Yokota, On the volume conjecture for hyperbolic knots, arXiv:math/0009165.

7. C. Zickert, The Chern-Simons invariant of a representation, arXiv:0710.2049. 
Department of Mathematical Sciences, Seoul National University, Seoul 151-742, Korea

E-mail address: jindol@math.snu.ac.kr

Department of Mathematics, Faculty of Science and Engineering, Waseda UniverSity, 3-4-1 Okubo, Shinjuku-Ku, Tokyo 169-8555, JAPAN

E-mail address: murakami@waseda.jp

Department of Mathematics, Tokyo Metropolitan University, Tokyo 192-0397, Japan

E-mail address: jojo@tmu.ac.jp 